\title{
Giant myxoma removal through a 3D-4K minimally invasive thoracoscopy: a case report and step-by-step guide
}

\author{
Francesco Pollari, Lucia Weber, Theodor Fischlein \\ Department of Cardiac Surgery, Klinikum Nürnberg - Paracelsus Medical University, Nuremberg, Germany \\ Correspondence to: Francesco Pollari, MD, PhD. Department of Cardiac Surgery, Klinikum Nürnberg - Paracelsus Medical University, Breslauer \\ Strasse 201, 90471 Nuremberg, Germany. Email: francesco.pollari@klinikum-nuernberg.de.
}

\begin{abstract}
The cardiac myxoma is a rare but potentially invalidating and fatal tumor, demanding for urgent surgery. The risk of intraoperative embolisms or incomplete removal of pedunculi, has been advocated as reasons for excluding patients from minimally invasive approach. However, this concept precludes the advantages of minimally invasive access to a category of patients which could profit from an early recovery, for example those with recent stroke. We aim to describe our positive experience in minimally invasive thoracotomy applied in a case of giant left atrial myxoma in an elderly and fragile woman. The patient was admitted in emergency department because of syncope. An echocardiogram revealed the presence of an intracardiac tumor that has been successfully removed with a mini-right thoracotomy. The postoperative course was uneventful and the patient was discharged on the $7^{\text {th }}$ postoperative day (POD). In this report, we aim to describe in detail step-to-step how to overcome eventually technical challenges and how to take full advantage of the latest surgical instruments for the minimally invasive approach. With a correct preoperative planning, synergistic preparation of the team and a deep knowledge of the tools used, the removal of a myxoma can be safely achieved, regardless of its size, as demonstrated in the illustrated case.
\end{abstract}

Keywords: Cardiac myxoma; cardiac tumor; minimally invasive; cardiac surgery; case report

Received: 18 June 2020; Accepted: 13 January 2021.

doi: 10.21037/jovs-20-136

View this article at: http://dx.doi.org/10.21037/jovs-20-136

\section{Introduction}

The cardiac myxoma is a benign tumor originating in the inside of cardiac chambers and so being a potential source of embolisms. Although rare [in a large autoptic study, $0.03 \%$ of subjects showed a primary cardiac tumor, in the $50 \%$ of which was a myxoma $(1,2)]$, it constitutes the most frequent primary tumor of the heart. The minimally invasive techniques in cardiac surgery have had a slow spread, despite outcome were comparable (if not superior) to traditional surgery. In particular, this approach has so far been used mainly for isolated pathologies (such as aortic or mitral valve disease), given the sense of insecurity derived from not having complete control in confined spaces, earning the fame of an underdog technique. However, not always the smaller means worst outcome or more strenuous working conditions. Moreover, the advantages of less traumatic procedure with the possibility of an earlier mobilization, could be an attractive option for many patients, especially those with neurological deficit and needing an early neurological rehabilitation. Despite some sporadic case reports already reported in the literature $(3,4)$, a detailed description on how to perform this difficult intervention (i.e., removal of a cardiac tumor) in a minimally invasive is missing. We aimed to show our minimally invasive cardiac surgery (MICS) technique that-as in a modern "David and Goliath" scenario-could successfully remove a giant left atrial myxoma. We present the following article in accordance with the CARE reporting checklist (available at http://dx.doi.org/10.21037/jovs-20-136).

\section{Case presentation}

A 65-year-old woman was admitted in our institution 
because of syncope. On the admission in emergency department, she was asymptomatic and showed no neurological deficits. The prior medical history was uneventful. She had no medications. A transthoracic echocardiography revealed a mass of circa $6 \mathrm{~cm}$ in left atrium. Following a cardiac magnetic resonance, the diagnosis of cardiac myxoma was confirmed.

The patient was driven in the standard pathway for every patient who is undergoing MICS. In this case, due to the urgency of the case, a faster organization has been performed in order to reduce the unpredictable risk of embolization. In 2 days from admission, the operation was scheduled, and the patient prepared after collection of informed consent. A contrast enhanced computer tomography was performed to assess the femoral vessel anatomy, aimed to ensure a peripheral circulation for cardiopulmonary bypass (CPB).

Our standard set for MICS was used. This consisted of: (I) a soft tissue retractor, (II) a $4 \mathrm{~K}$ and $3 \mathrm{D}$ videocamera (3D4K-VC) (Brown Einstein Vision 3.0, Aesculap, Germany), (III) external Scanlan ${ }^{\circledR}$ Chitwood clamp (Scanlan International, Inc., Saint Paul, MN 55107, USA), (IV) 3D glasses for operator and surgical assistant, (V) long instruments. Moreover, a C-curved long instrument (such as a tangential clamping forceps, e.g., Satinsky) for surrounding and lifting of the tumor was needed.

The $3 \mathrm{D}$ endoscope is connected through a $4 \mathrm{~K}$ HD camera head to a high-quality control unit. Two screens are used, one monitor for 2D standard vision (aimed to allow the surveillance of intervention by all surgical team, such as anesthesiologists and nurses) and one main ultra-3D-fullHD 32-inch screen optimized for 3D image reproduction. The three-dimensionality is very important for this procedure: not only compensate the possible reduction in view due to the minimal invasive access, it may also provide superior vision to traditional surgery.

The use of a dedicated venous cannula for right side operations is fundamental. In this case, as well as in minimally invasive mitral surgery, we use the "MICS Cannulae, RAP Femoral Venous, 22 Fr Distal/22 Fr Proximal, 200-100" (LivaNova). This cannula is designed for improved venous drainage, with a dual stage tip which drains from superior and inferior vena cava. As during extirpation of peduncoli a perforation of atrial septum occurs very oft (as also it can be seen in our experience, Video 1), the optimal venous drainage at the level of right atrium allows to ensure a rapid and efficient hemostasis. Retrograde perfusion and possible increase in $\mathrm{CPB}$ times during minimally invasive surgery
(MIS) can cause cerebral hypoperfusion. Moreover, at the removal of aortic clamp and reestablishment of normal cardiac pump activity, supraortic vessel could receive more desaturated blood from the heart rather than saturated blood from $\mathrm{CPB}$, resulting in a decrease of oxygen saturation in brain. To try to keep this possible complication under control in our center we use routinely the near-infrared spectroscopy (NIRS) to monitor constantly the brain saturation.

The CPB was established through femoral vessels. The cannulation of femoral artery and vein was performed with surgical cutdown (Video 2). After oblique incision of the groin, the femoral vessels were identified and exposed in their upper surface. Two concentric purse-string sutures with 5-0 polypropylene are applied on the vessels. After systemic heparinization, cannulation was performed using a standard Seldinger's technique. The venous cannula was inserted into the right femoral vein forwarded into the right atrium, under transesophageal echocardiography (TEE) guidance.

Hereafter, a mini-thoracotomy at $4^{\text {th }}$ right intercostal space was performed and a soft tissue retractor was used to retract the subcutaneous tissue and underlying muscle. A thoracic port was inserted cranially of the thoracic incision to allow the insertion of the endoscope for camera guidance. A transthoracic aortic clamp was used, introduced by a separate $5 \mathrm{~mm}$ incision in the midaxillary line. Antegrade cardioplegia was applied directly into the ascending aorta by a needle-vent catheter (Video 1). The left atrium was opened above the origin of the right upper pulmonary vein, as done in conventional mitral valve surgery. The tumor mass showed itself immediately after opening. Carefully dissection of peduncle was performed with curved scissors. The extraction of the tumor was achieved with a curved clamp. After removing of remains and burning of the previous tumor anchor point, left atrium was closed using a 4-0 polypropylene running suture. Before the suture was tied, careful antegrade and retrograde deairing was done through the atrium and the ascending aorta through the needle vent. Finally, wires for temporary pacing are placed.

The surgical team was composed by three surgeons (one operator and two assistant), a surgical nurse, a perfusionist and an anesthetist with experience in TEE. One of the two assistants was dedicated exclusively to the control of the video camera.

The patient was extubated in the intensive care unit in presence of stabile hemodynamic and no blood lose. Chest drains were removed on the $2^{\text {nd }}$ postoperative day (POD) and the patient was moved to the postoperative ward and 
discharged on the $7^{\text {th }}$ POD. At the outpatient check-up he was in excellent clinical condition, with no neurological signs or symptoms. A telephone follow-up 1 year after the procedure confirmed the good conditions, satisfaction of the patient for the fast recovery, without any recurrence of cardiovascular events.

All procedures performed in studies involving human participants were in accordance with the ethical standards of the institutional and/or national research committee(s) and with the Helsinki Declaration (as revised in 2013). Written informed consent was obtained from the patient.

\section{Discussion}

The two most important aspects for approaching myxomas removal in MIS are: (I) optimal vision of surgical field, especially of the peduncle; (II) the adequate instrument for tumor extraction.

The optimal vision is an indispensable issue of every surgical procedure, but in the present scenario has an adjunct value. The careful and meticulous removal of the peduncle is as important as removing of tumor itself, since an incomplete resection means a recurrence of myxomas growth. The reduced vision, or the feeling of, in MICS could be overcome through the use of the $3 \mathrm{D} 4 \mathrm{~K}-\mathrm{VC}$ as in the case presented. The most frequent pitfall is represented by the possible flooding of blood in the surgical field, due to the perforation of the atrial septum (during the removal of the peduncle) or the presence of a patent foramen ovale. In this case, the presence at the surgical table of an assistant dedicated to the governance and frequent cleaning of the video-camera, and another dedicated to the use of the aspirator, allows the operating surgeon to be able to react promptly and perform the appropriate hemostasis in a few seconds.

The second fundamental issue is the tumor extraction, that could be difficult especially in presence of a giant myxoma as here reported. The classical instruments for MIS are designed for repair or replacement of heart valves and are inadequate for this scope. Clinging the mass with forceps or Bengolea tools is not recommended, given the gelatinous consistency of these tumors and the tendency to crumble forming possible sources of emboli. The best solution, in our opinion, was to surround the mass with a curved instrument, like a Satinsky-clamp (Video 1).

\section{Conclusions}

Extirpation of a giant cardiac myxoma in mini-thoracotomy is a safe and viable treatment option when performed in an experienced minimal invasive cardiac surgery program. Our experience is in line with other similar cases recently reported $(3,4)$. We aimed to provide a step-to-step guide for those surgeons who want to supply their patients a less invasive procedure, keeping the safety and efficacy as well. Concerning about possible embolization or recidivism are understandable but could be overcome through right equipment and team-preparation and should not preclude the advantages of MICS.

\section{Acknowledgments}

Funding: None.

\section{Footnote}

Reporting Checklist: The authors have completed the CARE reporting checklist. Available at http://dx.doi.org/10.21037/ jovs-20-136

Conflicts of Interest: All authors have completed the ICMJE uniform disclosure form (available at http://dx.doi. org/10.21037/jovs-20-136). Prof. TF reports personal fees from LivaNova, outside the submitted work. And Prof. TF serves as an unpaid editorial board member of fournal of Visualized Surgery from Aug 2019 to Jul 2021. The other authors have no conflicts of interest to declare.

Ethical Statement: The authors are accountable for all aspects of the work in ensuring that questions related to the accuracy or integrity of any part of the work are appropriately investigated and resolved. All procedures performed in studies involving human participants were in accordance with the ethical standards of the institutional and/or national research committee(s) and with the Helsinki Declaration (as revised in 2013). Written informed consent was obtained from the patient.

Open Access Statement: This is an Open Access article distributed in accordance with the Creative Commons Attribution-NonCommercial-NoDerivs 4.0 International License (CC BY-NC-ND 4.0), which permits the noncommercial replication and distribution of the article with the strict proviso that no changes or edits are made and the original work is properly cited (including links to both the formal publication through the relevant DOI and the license). See: https://creativecommons.org/licenses/by-nc- 
nd/4.0/.

\section{References}

1. Frömke J. Standardoperationen in der Herzchirurgie. Heidelberg: Steinkopff-Verlag, 2003.

2. Reynen K. Cardiac myxomas. N Engl J Med

doi: $10.21037 /$ jovs-20-136

Cite this article as: Pollari F, Weber L, Fischlein T. Giant myxoma removal through a $3 \mathrm{D}-4 \mathrm{~K}$ minimally invasive thoracoscopy: a case report and step-by-step guide. J Vis Surg 2021.
1995;333:1610-7.

3. Gaisendrees C, Mader N, Sabashnikov A, et al. Minimally invasive resection of a giant left atrial myxoma: a case report. Perfusion 2020;35:263-6.

4. Dang QH, Le NT, Tran DD, et al. Totally endoscopic resection of giant left atrial myxoma without robotic assistance. Innovations (Phila) 2018;13:136-9. 